\title{
Broadband bright twin beams and their up-conversion
}

\author{
Maria V. Chekhova, ${ }^{1,2,3}$ Semen Germanskiy, ${ }^{3,4}$ Dmitri B. Horoshko, ${ }^{5,6}$ Galiya Kh. Kitaeva, ${ }^{3}$ \\ Mikhail I. Kolobov, ${ }^{5}$ Gerd Leuchs, ${ }^{1,2}$ Chris R. Phillips, ${ }^{7}$ and Pavel A. Prudkovskii ${ }^{3}$ \\ ${ }^{1}$ Max Planck Institute for the Science of Light, Staudtstraße 2, 91058 Erlangen, Germany \\ ${ }^{2}$ University of Erlangen-Nürnberg, Staudtstraße 7/B2, 91058 Erlangen, Germany \\ ${ }^{3}$ Department of Physics, M. V. Lomonosov Moscow State University, Leninskie Gory, 119991 Moscow, Russia \\ ${ }^{4}$ Helmholz Zentrum Dresden Rossendorf, Bautzner Landstr. 400, 01328 Dresden, Germany \\ ${ }^{5}$ Univ. Lille, CNRS, UMR 8523PhLAM F-59000 Lille, France \\ ${ }^{6}$ B. I. Stepanov Institute of Physics, NASB, Nezavisimosti Ave. 68, 220072 Minsk, Belarus \\ ${ }^{7}$ Department of Physics, ETH Zurich, CH-8093 Zurich, Switzerland
}

\begin{abstract}
We report on the observation of broadband (40 THz) bright twin beams through high-gain parametric down-conversion in an aperiodically poled lithium niobate crystal. The output photon number is shown to scale exponentially with the pump power and not with the pump amplitude, as in homogeneous crystals. Photon-number correlations and the number of frequency/temporal modes are assessed by spectral covariance measurements. By using sum-frequency generation on the surface of a non-phasematched crystal, we measure a cross-correlation peak with the temporal width 90 fs.
\end{abstract}

Within the broad subject of quantum state engineering, there is a lot of interest to the generation of ultrabroadband nonclassical light, in particular ultrabroadband or even single-cycle photon pairs (biphotons) 1 -4 4 . A broad spectrum in combination with a narrow correlation bandwidth means a high degree of frequency/time entanglement [5, 6] and can be used for high-dimensional quantum information encoding [7]. Moreover, the larger the bandwidth of an entangled source, the faster quantum communication it can provide [8]. Another motivation for engineering such sources is due to nonlinear optical effects with nonclassical light 99 11] and clock synchronization [12].

Biphotons with ultrabroad spectrum and a high degree of frequency entanglement can be obtained through parametric down-conversion (PDC) under frequencydegenerate phase matching at wavelengths with low group velocity dispersion 1, 13, 14], in a long crystal pumped by short pulses [5], by taking advantage of the whole frequency-angle spectrum of PDC [15, 16], or simply by using a very thin nonlinear crystal provided that the pumping is strong enough [17.

Another method to achieve an ultrabroad spectrum is to use aperiodically poled crystals $2-4,18-22]$. It enables not only broadening, but also engineering of the spectral shape in an arbitrary way, limited of course by the technology level. Another considerable advantage of this method is that it is principally lossless, in contrast to ones that involve diffractive or phase elements.

At the same time, the spectral broadening achieved through aperiodic poling is inhomogeneous, in the sense that photons at different wavelengths are generated at different positions in the crystal. For this reason, a phase chirp appears in the two-photon spectral amplitude, and reaching short correlation times requires its removal. Under inhomogeneous spectral broadening, the frequency dependence of the phase contains the squared phase mismatch both in the low-gain 2] and the highgain regimes 21. Thus the phase is a fourth-order polynomial of the frequency even in the simplest case of a quadratic dispersion dependence. For not very broad spectra the dominant quadratic phase chirp can be eliminated by using, for instance, a material with large groupvelocity dispersion (GVD) [2, 23]. However, this strategy does not work if the spectrum is broadened to values exceeding $10 \mathrm{THz}$ [21]. In this case, elimination of the phase chirp requires either active compensation or a special design of the crystal [21].

At strong pumping, PDC produces not biphotons but bright squeezed vacuum 24 27]: radiation with strong photon-number correlations and quadrature squeezing. Due to its high degree of photon-number entanglement 28], this quantum state of light has a high quantum information capacity, which can be further increased due to a large number of frequency modes. The ultimate bandwidth limit of the whole optical octave is attained for "single-cycle squeezed light" considered in [20].

In our experiment we generate bright squeezed vacuum in an aperiodically poled $5 \mathrm{~mm}$ long sample of lithium niobate $\left(\mathrm{LiNbO}_{3}\right)$ crystal doped with $\mathrm{MgO}$. The samples were produced by Gooch and Housego [29]. According to the design, the inverse grating vector should vary along the crystal as a squared hyperbolic function 21],

$$
K(z)=-\frac{\alpha}{4(2-z / L)^{2}}+\beta,
$$

with $\alpha=735 \mathrm{rad} / \mathrm{mm}, \beta=901 \mathrm{rad} / \mathrm{mm}$, and $z$ is the longitudinal coordinate (Fig. 1. A). Such a design provides compensation for the the third-order and fourth-order terms in the phase chirp without any additional optical elements. With the grating vector as shown in Fig. 1 $\mathrm{a}$, type- 0 phase matching condition is satisfied for pumping at $532 \mathrm{~nm}$, the signal and idler wavelengths being around $790 \mathrm{~nm}$ and $1600 \mathrm{~nm}$, respectively. 
The setup for the study of the spectral properties is shown in Fig. 10. The pump is the second-harmonic radiation of a Nd:YAG laser, producing 18-ps full-width at half maximum (FWHM) pulses at a wavelength of 532 nm, focused into the $\mathrm{LiNbO}_{3}$ crystal by lens L1 with the focal distance $500 \mathrm{~mm}$. The repetition rate is $1 \mathrm{kHz}$. The pump power in the crystal is up to $25 \mathrm{~mW}$, which corresponds to the peak intensity $0.51 \mathrm{GW} / \mathrm{cm}^{2}$, based on a focused beam size of $170 \mu \mathrm{m} 1 / e^{2}$ radius measured in air without the crystal.

After the crystal, the pump is cut off by a long-pass filter (LPF) and the signal and idler beams are separated by a dichroic mirror DM. In each beam, there is a lens (L2, L3), in whose focal plane an aperture selects nearly collinear emission: $5 \mathrm{mrad}$ and $2.5 \mathrm{mrad}$ for the idler and signal beams, respectively. After each aperture, the radiation is collected by lenses L4, L5 into a multimode fiber and fed into a spectrometer: AvaSpec-ULS3648 for the signal radiation and AvaSpec-NIRS12 for the idler radiation. The spectrometers are gated synchronously with the laser pulse.
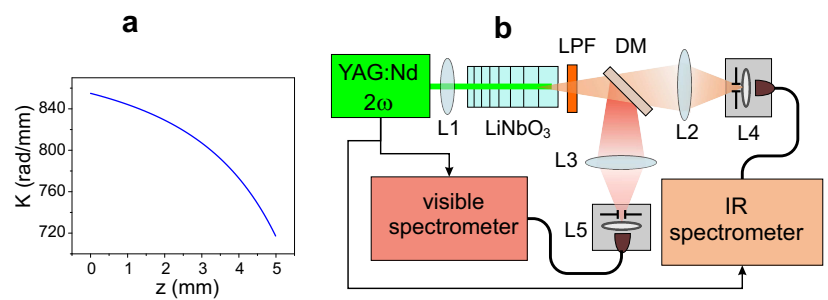

FIG. 1. The design of the aperiodic poling (a) and the setup for the study of spectral correlations (b).

The resulting spectra obtained for the signal and idler beams under pumping with the average power $10 \mathrm{~mW}$ are shown in Fig. 2a,b, respectively. The spectral sensitivities of both signal and idler arms of the setup were calibrated using the method described in Ref. [30], and the resulting spectra were corrected for this sensitivity. However, the NIR arm has zero sensitivity for wavelengths above $1650 \mathrm{~nm}$ (shown by shading); this is why the idler spectrum is cut on the 'red' side. At the same time, the spectrum of the signal beam is fully registered by the visible spectrometer, and the spectral width is $40 \mathrm{THz}$, or $90 \mathrm{~nm}$. The peaks observed in the spectra are caused by the non-idealities of the domain structure.

The spectrum expected for the ideal grating as shown in Fig. 17 can be calculated using the approach of Ref. [21]. We introduce the positive-frequency field for PDC light as

$$
E^{(+)}(t, z)=e^{-i \omega_{0} t} \int_{-\omega_{0}}^{\omega_{0}} \hat{b}(\Omega, z) e^{i\left\{k\left(\omega_{0}+\Omega\right) z-\Omega t\right\}} d \Omega
$$

where $\omega_{0}=\omega_{p} / 2$ is half the pump frequency and $\hat{b}(\Omega, z)$ the PDC photon annihilation operator at frequency $\omega_{0}+$
$\Omega$. Its evolution is given by the Bogolyubov transformation,

$$
\hat{b}(\Omega, z)=A(\Omega, z) \hat{b}(\Omega, 0)+B(\Omega, z) \hat{b}^{\dagger}(-\Omega, 0) .
$$

The evolution of $A(\Omega, z)$ and $B(\Omega, z)$ is described by the system of equations

$$
\begin{gathered}
\frac{\partial A(\Omega, z)}{\partial z}=i g B^{*}(\Omega, z) e^{i \Delta(\Omega) z-i \int_{0}^{z} K\left(z^{\prime}\right) d z^{\prime}} \\
\frac{\partial B^{*}(\Omega, z)}{\partial z}=-i g^{*} A(\Omega, z) e^{-i \Delta(\Omega) z+i \int_{0}^{z} K\left(z^{\prime}\right) d z^{\prime}}
\end{gathered}
$$

where $\Delta(\Omega)=k_{p}-k\left(\omega_{0}+\Omega\right)-k\left(\omega_{0}-\Omega\right)$ is the wavevector mismatch and $g$ the parametric interaction coefficient involving the pump field and the quadratic susceptibility. By numerically integrating this system, we obtain the spectrum of the signal beam $S(\Omega)=|B(\Omega, L)|^{2}$. Fig. $2 \mathrm{~d}$ shows this spectrum for the amplification coefficient $\nu_{0}=|g|^{2} L /|K(0)-K(L)|=0.1$. Compared to the theoretical prediction, the experimental spectrum is cut on the 'blue' side and especially on the 'red' side, probably due to the deviations of the $K(z)$ dependence from Eq.(11).
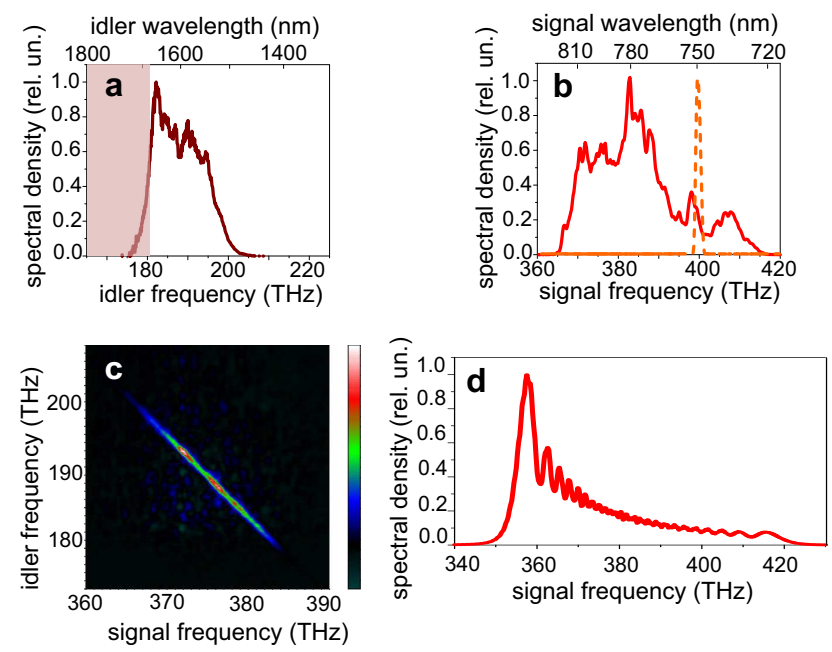

FIG. 2. Spectra of the signal and idler radiation emitted in the collinear direction $(\mathrm{a}, \mathrm{b})$ and the covariance describing the spectral correlations between them (c). Dashed orange line in (b) shows the spectrum of a periodically poled sample. Panel (d) shows the spectrum calculated according to the theory of Ref [21] with $\nu_{0}=0.1$.

The observed $40 \mathrm{THz}$ spectral width is far from being a record (compare, for instance, with Refs. [1, 16]), but is quite large for non-degenerate PDC. For comparison, panel (b) shows the signal spectrum obtained for a $\mathrm{LiNbO}_{3}$ crystal of the same length, poled with the constant grating vector $774 \mathrm{rad} / \mathrm{mm}$ (dashed orange line). The width is $1.3 \mathrm{THz}$, or $2.5 \mathrm{~nm}$, which shows that the aperiodic poling leads to the spectral broadening by a factor of 36 . 
To characterize the frequency correlations between the signal and idler beams, we take a set of 3000 single-pulse spectra and measure the covariance of signal and idler photon numbers as a function of frequencies $\omega_{s}, \omega_{i}$ 31, 32],

$$
\operatorname{Cov}\left(\omega_{s}, \omega_{i}\right)=\left\langle N\left(\omega_{s}\right) N\left(\omega_{i}\right)\right\rangle-\left\langle N\left(\omega_{s}\right)\right\rangle\left\langle N\left(\omega_{i}\right)\right\rangle .
$$

This distribution is shown in panel c of Fig. 2 and looks as a narrow stripe, which is an evidence of strong frequency correlations. Although the bottom-right part of the stripe is not visible due to the 'blindness' of the IR spectrometer in this range, one can still infer the number of modes using the ratio between the total spectral width $\Delta \omega$ and the width $\delta \omega$ of the covariance distribution cross-section [5],

$$
R=\Delta \omega / \delta \omega .
$$

The ratio (6) is 40, which is only the lower boundary of the number of modes, because Eq. (6) does not take into account possible additional entanglement in the phase of the two-photon amplitude.

The parametric gain is found from the dependence of the output signal after narrowband filtering on the pump power 27]. In contrast to the case of a homogeneous crystal, in an aperiodically poled one the effective length of nonlinear interaction scales linearly with the pump field amplitude [18, 21]. As a result, the number of photons per mode at high gain depends on the pump power $P$ as $N=A\left(e^{B P}-1\right)$, where $B$ is a parameter depending on the quadratic nonlinearity and its spatial modulation and $A \sim 1$. In other words, the parametric gain exponent $G=B P$ is a linear rather than square root function of the pump power. Using this dependence to fit the photon flux within a $5 \mathrm{~nm}$ bandwidth around $1600 \mathrm{~nm}$ as a function of the pump power (Fig. (3), we find that the parametric gain exponent under pumping at $15 \mathrm{~mW}$ is as high as $G=18$, resulting in $5 \cdot 10^{7}$ photons per mode. The same figure shows a fit by the $A \sinh ^{2}(B \sqrt{P})$ function, typical for high-gain PDC in a homogeneous crystal. The fit results in an unphysically small $A$ coefficient, which demonstrates its invalidity.

The linear rather than square-root dependence of the parametric gain exponent on the pump power has been found in the classical theory of parametric amplification in aperiodically poled crystals [18] and in the quantum theory of squeezed light generation in such crystals [20, 21]. It is generally known as the Rosenbluth gain, first obtained for a problem governed by the same differential equation in plasma physics 33]. It can be understood from a simple argument: the down-converted radiation around some frequency is generated in a layer of the crystal phase-matched for this frequency and has a gain proportional to the pump amplitude times the layer width. Theoretical analysis shows 21] that the width of the layer grows linearly with the pump amplitude; thus,

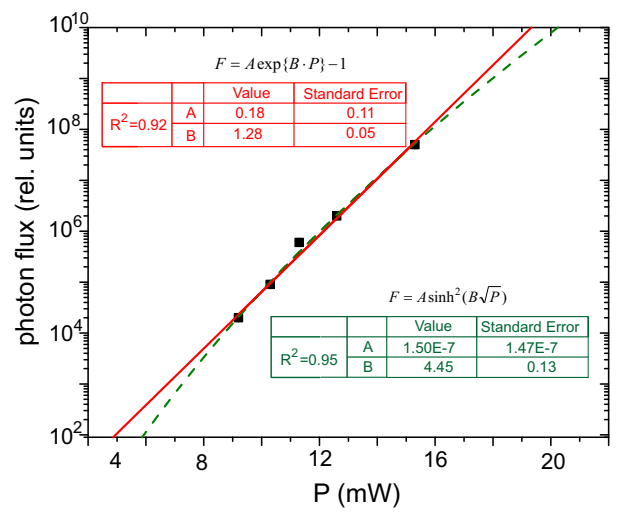

FIG. 3. Photon flux measured within a band of $5 \mathrm{~nm}$ at the wavelength $1600 \mathrm{~nm}$ as a function of the pump power. Red solid and green dashed lines are fits with different dependences.

the overall dependence of the gain is quadratic in the pump amplitude, or linear in its power.

Finally, the photon-number correlation time is measured with an ultrafast correlator shown in Fig. 4 The path length difference between the signal and idler beams is scanned by displacing a retroreflecting mirror (RM). After combining the signal and idler beams again on a dichroic mirror (DM), we focus them on the sumfrequency generation (SFG) crystal by means of a gold parabolic mirror. In contrast to previous work (see, for

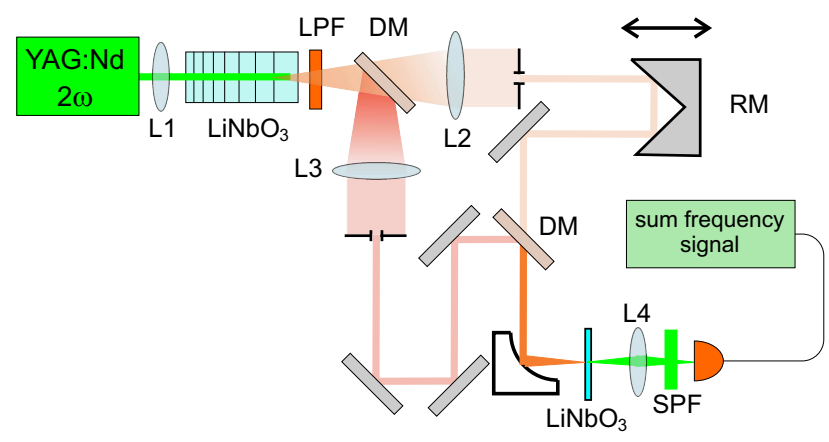

FIG. 4. The setup for measuring the temporal second-order intensity correlation function. Signal and idler beams, after a delay line, are combined on a dichroic mirror and focused on the surface of a lithium niobate crystal for sum-frequency generation.

instance, Ref. 8]) where phase matched SFG was used as a correlator, in our setup the SFG occurs without phase matching, on the surface of a $3 \mathrm{~mm}$ slab of $\mathrm{LiNbO}_{3}$ crystal. Due to the absence of phase matching, the SFG covers a broad spectral range and enables the measurement of ultra-short correlation times. Both signal and idler beams are polarized along the z-axis of the crystal, which provides a high efficiency of nonlinear conversion due to the large value of the quadratic susceptibility $\chi_{z z z}^{(2)}$. After the SFG, the signal and idler radiation is cut 
off by a short-pass filter (SPF) while the sum-frequency radiation around $532 \mathrm{~nm}$ is collected by lens L4 into a pulsed photon-number integrating detector based on a $\mathrm{p}-\mathrm{i}-\mathrm{n}$ diode. The power of the sum-frequency radiation is measured as a function of the time delay between the signal and the idler beams.

Fig 5 shows the results. Coarse scanning with a step of 1 ps (left panel) reveals the incoherent pedestal with the width determined by the pulse duration. The background is caused by the residual pump and signal radiation. Fine scanning around the top of the pedestal with a step of 10 fs (right panel) shows a 90 fs additional peak indicating ultrafast correlations of the twin beams. The reason for the pedestal being pronounced is the high brightness of the twin beams. A similar SHG time dependence was observed in Ref. 34 although with a much broader peak. At the same time, the 90 fs peak we measure is considerably narrower than the one observed for two-photon light generated by an aperiodically poled crystal [3].

The theoretical dependence of the SFG signal on the delay $\tau$ of the idler field with respect to the signal one can be obtained by writing the total PDC field at the input of the correlator as

$$
\begin{aligned}
E^{(+)}(t, L)= & e^{-i \omega_{0} t} \int_{0}^{\omega_{0}}\left[\hat{b}(\Omega, L) e^{i\left\{k\left(\omega_{0}+\Omega\right) L-\Omega t\right\}}+\right. \\
& \left.+\hat{b}(-\Omega, L) e^{i\left\{k\left(\omega_{0}-\Omega\right) L+\Omega(t+\tau)-\omega_{0} \tau\right\}}\right] d \Omega,(7)
\end{aligned}
$$

and by finding the mean intensity of the sum-frequency field,

$$
I_{S F G} \propto\left\langle\operatorname{vac}\left|\left[E^{(-)}(t, L)\right]^{2}\left[E^{(+)}(t, L)\right]^{2}\right| \operatorname{vac}\right\rangle .
$$

For a continuous-wave pump, we get the result similar to the one of Refs. [20, 35],

$$
\begin{array}{r}
I_{S F G}(\tau) \propto 8\left(\int_{0}^{\omega_{0}}|B(\Omega, L)|^{2} d \Omega\right)^{2}+ \\
+4\left|\int_{0}^{\omega_{0}} A(\Omega, L) B(\Omega, L) e^{i(\Omega \tau-\Delta(\Omega) L)} d \Omega\right|^{2} .
\end{array}
$$

The first term describes the non-coherent background, which in the case of pulsed pump is not constant but repeats the shape of the pulse auto-convolution, while the second term is the coherent component of SHG. Its width depends on the phase $\arg \{A(\Omega, L) B(\Omega, L)\}-\Delta(\Omega) L$. Calculation according to Eq. (9) (blue curve in Fig. 5) shows an asymmetric peak with the width similar to the one observed in the experiment.

The correlation time can be further reduced by compensating the quadratic phase chirp by using additional dispersive material.

In conclusion, we have observed the broadening of the spectrum of bright squeezed vacuum twin beams through the aperiodic poling of the $\mathrm{LiNbO}_{3}$ crystal. Compared to the periodically poled sample of the same length, the spectrum gets broadened by a factor of 36 . This is a
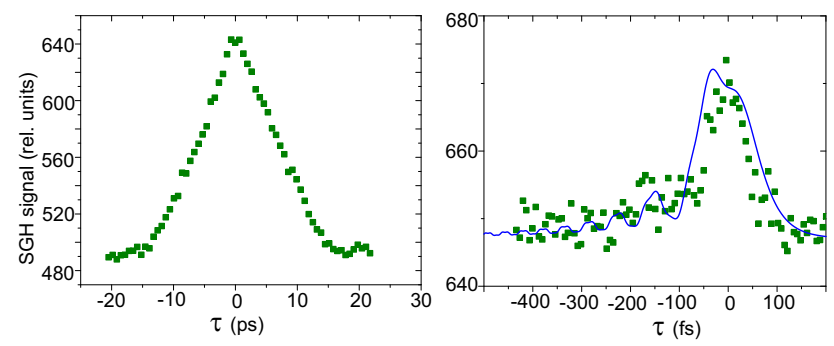

FIG. 5. Signal of sum frequency generation as a function of the time delay between the signal and idler beams. The left and right panels show the results of scanning with 1 ps and 10 fs resolution, respectively. The blue line shows the dependence calculated numerically for gain exponent $G=18$.

relatively large value, taking into account the fact that, theoretically, the spectrum should get narrower with a higher parametric gain.

The output photon number is shown to be exponentially increasing with the pump power according to the Rosenbluth law, typical for aperiodically poled crystals. This is different from the case of homogeneous crystals where the output photon number scales exponentially with the pump amplitude.

Measurement of the spectral covariance of the signal and idler beams revealed a large number of modes, their lower bound equal to 40 .

The use of aperiodic poling with the compensation of higher-order dispersion chirp, even without additional group-velocity dispersion elements, allowed us to observe a 90 fs broad peak in the temporal correlation function, which we have measured using an ultrafast correlator, based on the non-phasematched sum-frequency generation.

Apart from applications in quantum information, such bright twin beams with ultrashort correlation times will be very useful for nonlinear optics and spectroscopy.

\section{FUNDING INFORMATION}

This work was supported by the Russian Science Foundation grant No. 17-12-01134.

[1] Yaakov Shaked, Roey Pomerantz, Rafi Z. Vered and Avi Peer, New J. Phys. 16, 053012 (2014).

[2] S. E. Harris, Phys. Rev. Lett. 98, 063602 (2007).

[3] S. Sensarn, G. Y. Yin, and S. E. Harris, Phys. Rev. Lett. 104, 253602 (2010).

[4] Magued B. Nasr, Silvia Carrasco, Bahaa E. A. Saleh, Alexander V. Sergienko, Malvin C. Teich, Juan P. Torres, Lluis Torner, David S. Hum, and Martin M. Fejer, Phys. Rev. Lett. 100, 183601 (2008). 
[5] G. Brida, V. Caricato, M. V. Fedorov, M. Genovese, M. Gramegna, and S. P. Kulik, EPL 87, 64003 (2009).

[6] D. B. Horoshko, G. Patera, A. Gatti and M. I. Kolobov, Eur. Phys. J. D. 66, 239 (2012). 66, 239 (2012).

[7] B. Bessire, C. Bernhard, T. Feurer, and A. Stefanov, New J. Phys. 16, 033017 (2014).

[8] Barak Dayan, Avi Peer, Asher A. Friesem, and Yaron Silberberg, Phys. Rev. Lett. 94, 043602 (2005).

[9] D. N. Klyshko, Sov. Phys. JETP 56, 752 (1982).

[10] J. Gea-Banacloche, Phys. Rev. Lett. 62, 1603 (1989).

[11] N. Georgiades, E. S. Polzik, K. Edamatsu, H. J. Kimble, A. S. Parkins, Phys. Rev. Lett. 75, 3426 (1995).

[12] A. Valencia, G. Scarcelli, and Y. Shih, Appl. Phys. Lett. 85, 2655 (2004).

[13] D. Strekalov, A. B. Matsko, A. A. Savchenkov, and L. Maleki, J. Mod. Opt. 52, 2233 (2005).

[14] Kevin A. O'Donnell and Alfred B. U'Ren, Opt. Lett. 32, 817-819 (2007).

[15] Barak Dayan, Avi Peer, Asher A. Friesem, and Yaron Silberberg, Phys. Rev. Lett. 93, 023005 (2004).

[16] K. G. Katamadze, N. A. Borshchevskaya, I. V. Dyakonov, A. V. Paterova, and S. P. Kulik, Phys. Rev. A 92, 023812 (2015).

[17] K. G. Katamadze, N. A. Borshchevskaya, I. V. Dyakonov, A. V. Paterova, and S. P. Kulik, Laser Phys. Lett. 10, 045203 (2013).

[18] M. Charbonneau-Lefort, B. Afeyan, and M. M. Fejer, J. Opt. Soc. Am. B 25, 463 (2008).

[19] M. Charbonneau-Lefort, B. Afeyan, and M. M. Fejer, J. Opt. Soc. Am. B 25, 680 (2008).

[20] D. B. Horoshko and M. I. Kolobov, Phys. Rev. A 88, 033806 (2013).
[21] D. B. Horoshko and M. I. Kolobov, Phys. Rev. A 95, 033837 (2017).

[22] C. R. Phillips, B. W. Mayer, L. Gallmann, M. M. Fejer, and U. Keller, Opt. Express 22, 9627 (2014).

[23] G. Brida, M. V. Chekhova, I. P. Degiovanni, M. Genovese, G. Kh. Kitaeva, A. Meda, and O. A. Shumilkina, Phys. Rev. Lett. 103, 193602 (2009).

[24] O. Jedrkiewicz, Y.-K. Jiang, E. Brambilla, A. Gatti, M. Bache, L. A. Lugiato, and P. Di Trapani, Physical Review Letters 93, 243601 (2004).

[25] M. Bondani, A. Allevi, G. Zambra, M. G. A. Paris, and A. Andreoni, Physical Review A 76, 013833 (2007).

[26] G. Brida, L. Caspani, A. Gatti, M. Genovese, A. Meda, and I. R. Berchera, Physical Review Letters 102, 213602 (2009).

[27] I. N. Agafonov, M. V. Chekhova, and G. Leuchs, Phys. Rev. A 82, 011801 (2010).

[28] M. V. Chekhova, G. Leuchs, M. Zukowski, Optics Commun. 337, 27-43 (2015).

[29] https://goochandhousego.com/

[30] S. Lemieux, M. Manceau, P. R. Sharapova, O. V. Tikhonova, R. W. Boyd, G. Leuchs and M. V. Chekhova, Phys. Rev. Lett. 117, 183601 (2016).

[31] K. Yu. Spasibko, T. Sh. Iskhakov, and M. V. Chekhova, Opt. Express 20, 7507 (2012).

[32] M. A. Finger, N. Y. Joly, P. St.J. Russell, M. V. Chekhova, Phys. Rev. A 95, 053814 (2016).

[33] M. N. Rosenbluth, Phys. Rev. Lett. 29, 565 (1972).

[34] I. Abram, R. K. Raj, J. L. Oudar, and G. Dolique, Phys. Rev. Lett. 57, 2516 (1986).

[35] B. Dayan, Phys. Rev. A 76, 043813 (2007). 